\title{
The effect of reported high-velocity small raindrops on inferred drop size distributions and derived power laws
}

\author{
H. Leijnse ${ }^{1, *}$ and R. Uijlenhoet ${ }^{1}$ \\ ${ }^{1}$ Hydrology and Quantitative Water Management Group, Department of Environmental Sciences, Wageningen University, \\ Droevendaalsesteeg 4, 6708 PB Wageningen, The Netherlands \\ * current address: Royal Netherlands Meteorological Institute, Wilhelminalaan 10, 3732 GK De Bilt, The Netherlands
}

Received: 2 February 2010 - Published in Atmos. Chem. Phys. Discuss.: 9 April 2010

Revised: 16 July 2010 - Accepted: 19 July 2010 - Published: 23 July 2010

\begin{abstract}
It has recently been shown that at high rainfall intensities, small raindrops may fall with much larger velocities than would be expected from their diameters. These were argued to be fragments of recently broken-up larger drops. In this paper we quantify the effect of this phenomenon on raindrop size distribution measurements from a Joss-Waldvogel disdrometer, a 2-D Video Distrometer, and a vertically-pointing Doppler radar. Probability distributions of fall velocities have been parameterized, where the parameters are functions of both rainfall intensity and drop size. These parameterizations have been used to correct JossWaldvogel disdrometer measurements for this phenomenon. The effect of these corrections on fitted scaled drop size distributions are apparent but not major. Fitted gamma distributions for three different types of rainfall have been used to simulate drop size measurements. The effect of the highvelocity small drops is shown to be minor. Especially for the purpose of remote sensing of rainfall using radar, microwave links, or optical links, the errors caused by using the slightly different retrieval relations will be masked completely by other error sources.
\end{abstract}

\section{Introduction}

It has recently been shown that in heavy rain, small drops do not all travel at (or even near) their theoretical terminal fall velocity (Montero-Martínez et al., 2009). This deviation was explained by the hypothesis that these drops are the result of

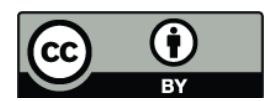

Correspondence to: H. Leijnse

(hidde.leijnse@knmi.nl) a recent break-up of a larger drop, which travels at a much greater velocity (e.g. Beard, 1976). Montero-Martínez et al. (2009) state in their conclusions that this deviation may have severe effects on drop size distributions (DSDs) derived from Joss-Waldvogel disdrometer (JWD), 2-D Video Distrometer (2DVD), and vertically-pointing Doppler radar measurements, and the subsequently derived radar reflectivityrainfall intensity relations.

In this paper, we do not focus on the origin of the reported high-velocity raindrops, but we attempt to quantify the severity of the mentioned effects, taking the observations of Montero-Martínez et al. (2009) as starting point. This will be done by simulating disdrometer measurements using a known drop size distribution, in a manner similar to that of Salles and Creutin (2003). Besides the effect on relations between the rainfall intensity $R$ and the radar reflectivity factor $Z$ (e.g. Battan, 1973; Doviak and Zrnic, 1993), we will also investigate the effect on relations between $R$ and other remote-sensing variables derived from these disdrometer measurements, namely the specific attenuation at $27 \mathrm{GHz}$ $k_{27}$ (Leijnse et al., 2007b), and the specific optical extinction $k_{\text {opt }}$ (Uijlenhoet et al., 2010).

To simulate disdrometer measurements, it is necessary to quantify the probability distribution of velocities for a given drop size at a given rainfall intensity. To this end, an attempt is made to parameterize the distributions of fall velocities as given by Montero-Martínez et al. (2009) in Sect. 2 . In Sect. 3, a method to correct JWD data for this phenomenon is proposed and applied to data from a squall line with three contrasting rainfall regimes (convective, transition, and stratiform) (Uijlenhoet et al., 2003). Resulting corrected data are normalized according to the scaling-law formalism (Sempere Torres et al., 1994), and exponential and

Published by Copernicus Publications on behalf of the European Geosciences Union. 
gamma distributions are fitted to these. Disdrometer measurements will then be simulated using these gamma distributions and the parameterizations of the distribution of velocity ratios in Sect. 4, where a Joss-Waldvogel disdrometer (Joss and Waldvogel, 1967), a 2-D Video Distrometer (Kruger and Krajewski, 2002), and a vertically-pointing Doppler radar (Doviak and Zrnic, 1993; Löffler-Mang et al., 1999) are considered. The resulting systematic errors resulting from the phenomenon described by Montero-Martínez et al. (2009) will be compared to errors resulting from other effects such as vertical wind (e.g. Salles and Creutin, 2003). The effect on power-law rainfall retrieval relations is investigated in Sect. 5. Finally, the conclusions of this study are presented in Sect. 6.

\section{Statistics of raindrop fall velocities}

The size distribution of raindrops is generally expressed as the density of drops of a given diameter class in a given volume (e.g. Marshall and Palmer, 1948). In order to be consistent with this definition, we would like to express the distribution of the fall velocities of drops in a similar form (i.e. in a volume). The probability density functions (pdf) of the ratio of actual $(v)$ and theoretical $\left(v_{t}\right)$ fall velocities presented in Fig. 2b of Montero-Martínez et al. (2009) is a pdf of the velocities of drops falling through a certain horizontal area over a given time interval. This pdf is related to the pdf of fall velocities in a given volume through the fall velocity itself (e.g. Uijlenhoet and Stricker, 1999). It could be argued that the velocity that should be used to convert the part of the pdf that is influenced by breakups is that of the large drops before breakup. Here, we will use the actual fall velocity to convert the area-pdf to a volume-pdf because it is difficult to determine the size of the original large drop. This approach will provide an upper limit to the effect of enhanced speed with respect to using the velocity of the original large drop.

The probability density functions of velocity ratios $x$ of drops of a given diameter falling through a horizontal area $f_{A}(x)$ and in a volume $f_{V}(x)$ are related as follows

$$
f_{A}(x)=\frac{x f_{V}(x)}{\int_{0}^{\infty} x f_{V}(x) \mathrm{d} x}=\frac{x f_{V}(x)}{\mu_{x V}},
$$

where $\mu_{x V}$ is the mean of $x$ of drops in a volume. The mean of $f_{A}(x)$ (i.e. $\left.\mu_{x A}\right)$ is related to the moments of $f_{V}(x)$ through

$\mu_{x A}=\int_{0}^{\infty} x f_{A}(x) \mathrm{d} x=\frac{1}{\mu_{x V}} \int_{0}^{\infty} x^{2} f_{V}(x) \mathrm{d} x$,

which is the ratio of the second and first moments of $f_{V}(x)$. This $\mu_{x A}$ is the variable that is plotted in Fig. 2a of MonteroMartínez et al. (2009), where it is called $\bar{v} / v_{t}$.

We assume the pdf of the ratio of the true drop velocity to the theoretical drop velocity $x$ to have two parts. One part

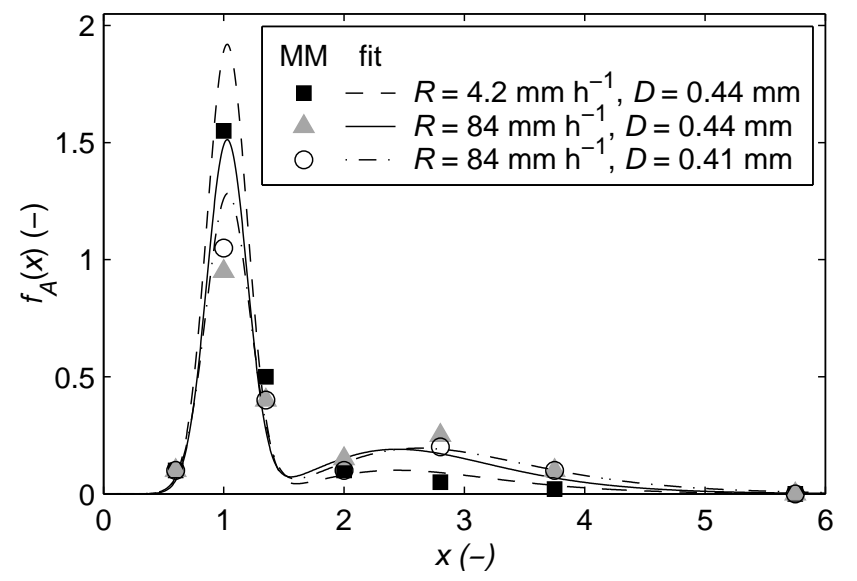

Fig. 1. Probability density functions (pdfs) of the ratio of fall velocities $f_{A}(x)$ compared to the pdfs shown in Fig. 2b of MonteroMartínez et al. (2009) (MM in legend), for different drop sizes and rainfall intensities. Note that Fig. 2b of Montero-Martínez et al. (2009) (and hence the symbols in this graph that are derived from this) do not represent true pdfs, as the area below these graphs is not unity.

is related to the drop fall velocities as we expect them to be (i.e. $v / v_{t}=1$ ), and the other part is related to the velocities of small drops that have resulted from a breakup which occurred less than their relaxation time (i.e. time between breakup and that required to reach their terminal velocities) ago. Because we would like to investigate the effect of the severe velocity underestimation by a theoretical relation, instead of the effect of vertical wind, we will assume that the first part is a monodisperse distribution. The second part of the distribution is assumed to be a log-normal distribution (e.g. Mood et al., 1974), of which the moments can be easily expressed by $\mathrm{E}\left[x^{n}\right]=\exp \left[n \mu_{\ln (x)}+\frac{1}{2} n^{2} \sigma_{\ln (x)}^{2}\right]$. The resulting distribution is given by

$$
f_{V}(x)=\alpha \delta(x-1)+\frac{1-\alpha}{x \sigma_{\ln (x)} \sqrt{2 \pi}} \exp \left[-\frac{\left(\ln (x)-\mu_{\ln (x)}\right)^{2}}{2 \sigma_{\ln (x)}^{2}}\right],
$$

where $\alpha$ is a parameter controlling the fraction of drops that have a velocity equal to the theoretical fall velocity $v_{t}$, and $\mu_{\ln (x)}$ and $\sigma_{\ln (x)}$ are parameters controlling the fall velocities of drops resulting from breakups of drops of a given size. To reduce the number of parameters in the given pdf, we will assume that $\sigma_{\ln (x)}^{2}=\beta \mu_{\ln (x)}$. Based on inspection of Fig. 2b of Montero-Martínez et al. (2009) (see Fig. 1), we estimate the value of $\beta$ to be approximately 0.1 .

The remaining parameters, $\alpha$ and $\mu_{\ln (x)}$, will of course depend on either the considered drop diameter $D$ or the rainfall intensity $R$, or on both. We will estimate the parameter $\mu_{\ln (x)}$ based on the observation that the mean of the velocities of fragments of drops in a volume should be between that of large drops (before break-up) and the theoretical terminal 


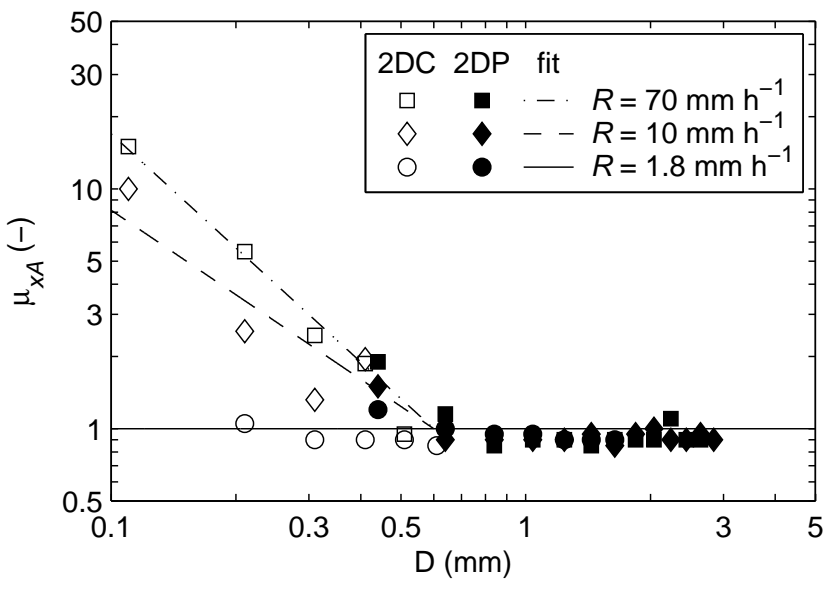

Fig. 2. Fits of relations between $D$ and $\mu_{x A}$ for different rainfall intensities to data from Fig. 2a of Montero-Martínez et al. (2009), with 2-DC and 2-DP indicating the different instruments that were used.

fall velocities of the fragments. We will assume here that this mean velocity should be approximately half of that of large drops, which we assume to be $v_{\max }=9 \mathrm{~m} \mathrm{~s}^{-1}$ (e.g. Beard, 1976). This mean velocity is larger than the theoretical terminal fall velocities of drops with diameters smaller than $1 \mathrm{~mm}$. This yields a mean of the lognormal part of $f_{A}(x)$ of 2.79. Figure 1 shows that the resulting $f_{A}(x)$ corresponds nicely with the distribution in Fig. $2 b$ of Montero-Martínez et al. (2009), where the part related to the high-velocity small drops has a mean between approximately 2.5 and 3 . The parameter $\mu_{\ln (x)}$ can be computed using

$\mu_{\ln (x)}=\frac{1}{1+\frac{1}{2} \beta} \ln \left(\frac{v_{\max }}{2 v_{t}(D)}\right)$.

The relation between the parameter $\alpha$ and $\mu_{x A}$ (the quantity given in Fig. 2a of Montero-Martínez et al., 2009) can be derived using Eqs. (2) and (3).

$\alpha=\frac{\mu_{x A} \mathrm{E}[x]-\mathrm{E}\left[x^{2}\right]}{\mu_{x A} \mathrm{E}[x]-\mathrm{E}\left[x^{2}\right]-\mu_{x A}+1}$,

where

$$
\begin{aligned}
& \mathrm{E}[x]=\exp \left[\left(1+\frac{1}{2} \beta\right) \mu_{\ln (x)}\right] \\
& \mathrm{E}\left[x^{2}\right]=\exp \left[(2+2 \beta) \mu_{\ln (x)}\right] .
\end{aligned}
$$

The dependence of the parameter $\alpha$ on $D$ and $R$ is computed by determining the relations between $\mu_{x A}$ (or $\bar{v} / v_{t}$ ) and $D$ and $R$ from Fig. 2a of Montero-Martínez et al. (2009). We have assumed a power-law dependence between $\mu_{x A}$ and $D$, with the coefficient determined such that $\mu_{x A}=1$ for $D=$
$0.6 \mathrm{~mm}$. The exponent is related to $R$ through another power law. The resulting function is given by

$\mu_{x A}= \begin{cases}\left(\frac{D}{D_{1}}\right)^{a_{\mu}\left(R-R_{1}\right)^{b} \mu} & \text { if } R>R_{1} \wedge D<D_{1} \\ 1 & \text { if } R \leq R_{1} \bigvee D \geq D_{1},\end{cases}$

where $D_{1}=0.6 \mathrm{~mm}$ and $R_{1}=2.5 \mathrm{~mm} \mathrm{~h}^{-1}$. Figure 2 shows the results of these fits. The dependence of the slopes of these lines on $R$ can be derived deterministically, as there are only two data points (slopes of the $R=10 \mathrm{~mm} \mathrm{~h}^{-1}$ and $R=$ $70 \mathrm{~mm} \mathrm{~h}^{-1}$ lines) to fit a two-parameter $\left(a_{\mu}\right.$ and $\left.b_{\mu}\right)$ relation. The resulting parameters are $a_{\mu}=-0.8892\left(\mathrm{~mm} \mathrm{~h}^{-1}\right)^{-b \mu}$ and $b_{\mu}=0.1365$.

It is clear from Fig. 2b of Montero-Martínez et al. (2009) (see also Fig. 1) that the monodisperse part of the distribution (i.e. the Dirac delta function) is different from the actual velocity distribution, which looks more like a normal distribution than a Dirac delta function. This is probably due to drop velocity variations due to fluctuations in vertical air velocity. However, because we would like to quantify the effect of the fragmentation phenomenon described by Montero-Martínez et al. (2009), the monodisperse distribution will be employed as a limiting case nonetheless. To investigate the relative importance of vertical wind variations we will also consider a distribution where the the Dirac delta function in Eq. (3) has been replaced by a normal distribution with mean 1 and standard deviation $\sigma=\sigma_{v} / v_{t}(D)$. We assume that the variations in vertical drop velocity due to vertical air velocity fluctuations do not depend on drop size (but $\sigma$ does through $v_{t}(D)$ ). This is based on the assumption that all drops are passive tracers of the wind field, the validity of which will decrease with drop size. However, insufficient information is available to derive relations between $\sigma_{v}$ and $D$. We have derived the value of $\sigma_{v}=0.3 \mathrm{~m} \mathrm{~s}^{-1}$ from Fig. 2b of Montero-Martínez et al. (2009) (see also Fig. 1). Note that this value is rather small compared to the values reported by Salles and Creutin (2003), i.e. $\sigma_{v} \geq 0.6 \mathrm{~m} \mathrm{~s}^{-1}$. This is probably due to the restriction to calm conditions (wind speeds $\leq 2 \mathrm{~m} \mathrm{~s}^{-1}$ ) of the data used by Montero-Martínez et al. (2009). The same normal distribution without the lognormal part of the distribution is used as a reference. The theoretical fall velocity corresponds to the assumption of a monodisperse velocity ratio distribution at 1 . This last distribution will also be used as a reference. The four distributions used are listed in Table 1, along with labels ((i) through (iv)) used in the remainder of this paper.

\section{Correction for Joss-Waldvogel disdrometer measurements}

In the present and subsequent sections, we will analyse the effect of the reported high-speed small raindrops on disdrometer measurements. The analyses will first be focused on the 
Table 1. Distributions of velocity ratios used throughout this paper, with the corresponding assumptions regarding the presence of the effects of high-speed small drops and turbulence-induced variations in fall velocities.

\begin{tabular}{lll}
\hline label & distribution & assumptions \\
\hline (i) & monodisperse distribution & no high-speed small drops, no turbulence \\
(ii) & normal distribution & no high-speed small drops, turbulence \\
(iii) & monodisperse + lognormal distribution & high-speed small drops, no turbulence \\
(iv) & normal + lognormal distribution & high-speed small drops, turbulence \\
\hline
\end{tabular}

effect on derived DSDs, and later on the power-law rainfall retrieval relations derived from these DSDs used in remote sensing. The three remote sensing variables that will be considered in this paper are the radar reflectivity factor $Z$ $\left(\mathrm{mm}^{6} \mathrm{~m}^{-3}\right)$, the specific attenuation of a $27-\mathrm{GHz}$ microwave signal $k_{27}\left(\mathrm{~dB} \mathrm{~km}^{-1}\right)$, and the specific optical extinction $k_{\mathrm{opt}}$ $\left(\mathrm{dB} \mathrm{km}^{-1}\right)$. These bulk rainfall variables and the rainfall intensity are determined from the drop size distribution $N_{V}(D)$ $\left(\mathrm{mm}^{-1} \mathrm{~m}^{-3}\right)$ according to

$$
\begin{aligned}
R & =6 \pi \times 10^{-4} \int_{0}^{D_{\max }} v_{t}(D) D^{3} N_{V}(D) \mathrm{d} D \\
Z & =\int_{0}^{D_{\max }} D^{6} N_{V}(D) \mathrm{d} D \\
k_{27} & =\frac{0.01}{\ln (10)} \int_{0}^{D_{\max }} Q_{\text {ext }}(D) N_{V}(D) \mathrm{d} D \\
k_{\mathrm{opt}} & =\frac{0.01 \pi}{2 \ln (10)} \int_{0}^{D_{\max }} D^{2} N_{V}(D) \mathrm{d} D,
\end{aligned}
$$

where $Q_{\text {ext }}(D)\left(\mathrm{mm}^{2}\right)$ is the extinction cross-section of a drop at $27 \mathrm{GHz}$, which can be computed using the T-matrix method (e.g. Mishchenko, 2000). Simulated DSDs are truncated at $D_{\max }=5.5 \mathrm{~mm}$ for computing $R, Z, k_{27}$, and $k_{\mathrm{opt}}$, as this is the maximum drop diameter measured by the JossWaldvogel disdrometer (JWD).

A Joss-waldvogel disdrometer (JWD) measures raindrop sizes through their impact on a styrofoam cone. The signal $U$ caused by a raindrop falling on the JWD is a function of raindrop diameter and fall velocity through

$U \sim D^{a} v^{b}$.

$U$ is effectively the result of a combination of 1) the momentum ( $a=3$ and $b=1) ; 2$ ) the kinetic energy ( $a=3$ and $b=2)$; and 3) the peak of the impact $(a=2$ and $b=2)$ of the drop. If we assume that Eq. (13) and the values of $a$ and $b$ hold perfectly, the diameter estimated by the JWD ( $\left.D_{\mathrm{JWD}}\right)$ is related to the true diameter through (Salles and Creutin, 2003)

$D_{\mathrm{JWD}}=D\left(\frac{v}{v_{t}}\right)^{b / a}$.

Joss and Waldvogel (1977) state that the empirical powerlaw relation between $U$ and $D$ will vary with the drop diameter, with the largest values of the exponent (4.3) occurring at small raindrops. Using the power-law approximation to the $v_{t}(D)$ relation by Atlas and Ulbrich (1977) (i.e. $v \sim D^{0.67}$ ), we can conclude that for small drops (the main focus of this paper) $U$ is mostly a function of kinetic energy ( $a=3$ and $b=2$, yielding an exponent of 4.34 for the $U-D$ relation). We will therefore use these values of $a$ and $b$ in subsequent analyses.

\subsection{Correction method}

Given the velocity ratio distributions derived in Sect. 2, it is possible to correct for the effect of high-velocity small drops in DSD measurements. Here, we focus on correcting DSDs measured by a JWD. The JWD has 20 diameter classes between $0.3 \mathrm{~mm}$ and $5.5 \mathrm{~mm}$, with class widths of $0.1 \mathrm{~mm}$ for diameters below $D_{1}=0.6 \mathrm{~mm}$ (the diameter above which we assume all drops to fall at their theoretical terminal fall velocity, see Sect. 2, Fig. 2, and Fig. 2a of Montero-Martínez et al., 2009). The contribution of drops with diameters within a given diameter class will, because of modified velocities, contribute to all of the diameter classes. If we denote the true numbers of drops per diameter class by the vector $N$, and those measured by the JWD as $N_{\text {JWD }}$, the relation between the two can be expressed by

$N_{\mathrm{JWD}}=A N$,

where $\boldsymbol{A}$ is a matrix with elements $A_{i j}$ containing the weights of the different contributions of drops of class $i$ to JWDmeasured drops of class $j$. This can easily be inverted

$N=A^{-1} N_{\text {JWD }}$.

Because the correction scheme depends on the rainfall intensity $R$ (see Eq. 8), and the corrected DSD will yield a different $R$ than the original, iteration of the method is required. In this iteration the rainfall intensity is varied until it converges to the $R$ resulting from the corrected DSD.

It should be noted that the correction method of Eq. (16) could potentially yield negative drop concentrations. These negative concentrations are set to zero. Correcting for highspeed small drops can therefore lead to higher values of bulk rainfall variables.

Because the diameter classes have finite widths, it is not known how drop diameters are distributed within this class. We therefore compute $\boldsymbol{A}$ in three different ways, (1) the drops 

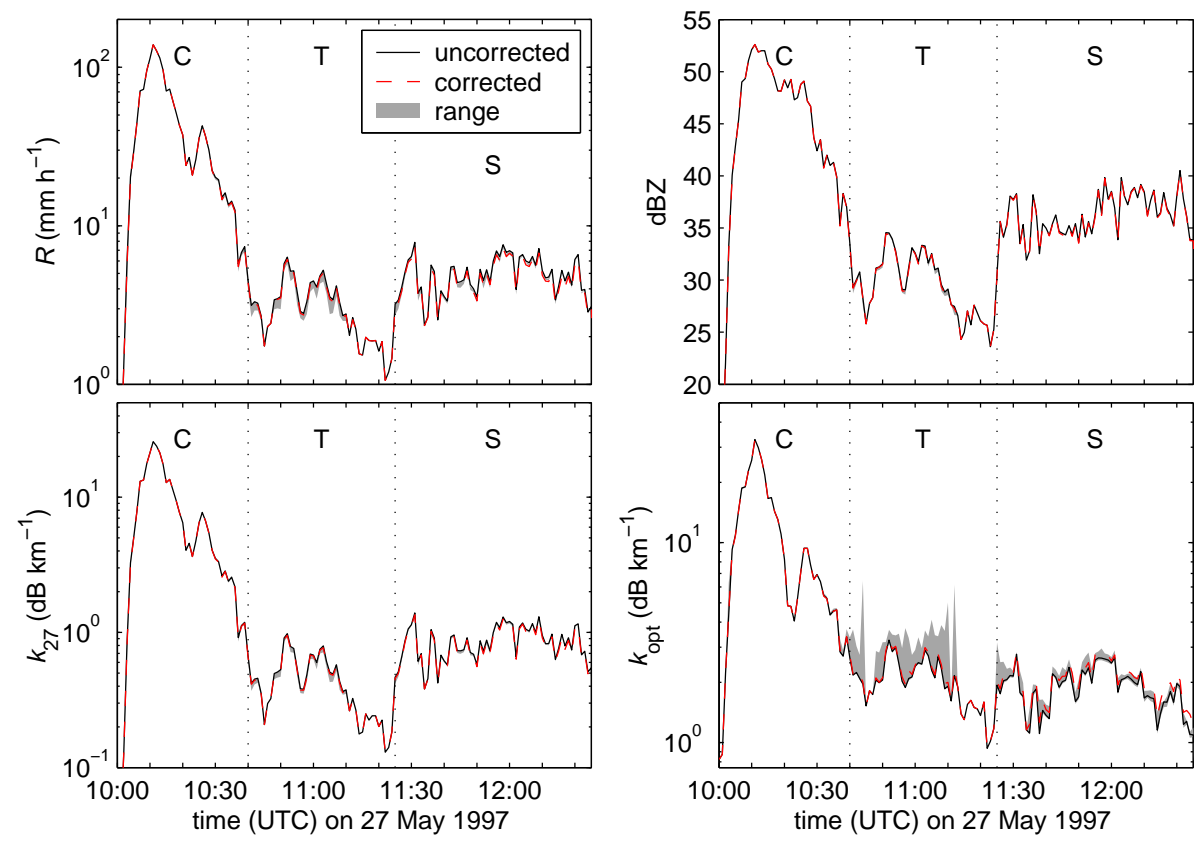

Fig. 3. Timeseries of bulk rainfall variables $\left(R, Z, k_{27}\right.$, and $\left.k_{\mathrm{opt}}\right)$ derived from the Goodwin Creek JWD dataset for the event of May 27 , 1997, with the convective (C), transition (T), and stratiform (S) regions (Uijlenhoet et al., 2003) indicated. The variables are computed from uncorrected and corrected DSDs, where the three methods of correcting described in Sect. 3.1 are depicted. Method (1) corresponds to the dashed red line (corrected), and the range between methods (2) and (3) is depicted by the shaded ares (range).

are assumed to be uniformly distributed in each diameter class, (2) all drops are assumed to have diameters equal to the lower limit of their corresponding class, and (3) all drops are assumed to have diameters equal to the upper limit of their corresponding class. Methods (2) and (3) are hence limiting cases, which will yield a band of possible corrections. It should be noted that finite diameter class widths of any disdrometer will cause uncertainties in any analysis carried out with disdrometer data because of precisely this reason.

\subsection{Application to JWD data}

The correction method proposed above is applied to data collected in a rainfall event on 27 May 1997 in the Goodwin Creek experimental watershed in northern Mississippi. These data have been thoroughly analyzed by Uijlenhoet et al. (2003), who have divided the dataset into three periods: convective $(\mathrm{C})$, transition $(\mathrm{T})$, and stratiform $(\mathrm{S})$. Figure 3 shows the effect of the corrections described in Sect. 3.1 applied to four bulk rainfall variables for the event on May 27, 1997. To show the effect of the different assumptions regarding the distribution of drop diameters within diameter classes on the correction method, the range between correction methods (2) and (3) is shown as a shaded area. It is clear from Fig. 3 that for $R, k_{27}$, and $Z$, the largest part of the band is generally below the line corresponding to a uniform distribution of drop sizes within each class. For $k_{\mathrm{opt}}$, the largest part of the band is above it. Because $k_{\mathrm{opt}}$ is a low order moment ( $2^{\text {nd }}$ order) of the DSD, it is very sensitive to the concentration of small drops. If all drops are assumed to be at the lower edge of the drop size class, the correction may yield a much larger number of small drops, whereas the correction may not have any effect if all drops are assumed to be at the upper edge of the class. This is particularly the case for low rainfall intensities (but above $2.5 \mathrm{~mm} \mathrm{~h}^{-1}$ ) because (1) small drops generally dominate at these intensities, and (2) the effect of the correction may be limited so that the difference between the assumed distributions within drop classes is largest. It is clear from this figure that the correction has very little effect on all bulk rainfall variables considered. The effect of the different assumptions regarding the distribution of drops within each diameter class is only important for specific optical extinction $k_{\mathrm{opt}}$ in the stratiform and especially the transition regions of the event. This is to be expected as $k_{\text {opt }}$ depends more on small drops than the other bulk variables considered. In the analyses presented in the remainder of this paper we will therefore only consider JWD data corrected using method (1).

As in Uijlenhoet et al. (2003), we will apply the scalinglaw formalism proposed by Sempere Torres et al. (1994) to these DSDs. In short, the drop size distribution $N_{V}(D)$ is expressed as

$$
N_{V}(D)=\Psi^{\alpha} g\left(\Psi^{-\beta} D\right),
$$


Table 2. Results of scaling analyses performed on the Goodwin Creek dataset for the three different regions within the event: convective (C); transition (T); and stratiform (S). Results are shown for corrected and uncorrected (Uijlenhoet et al., 2003) JWD data.

\begin{tabular}{|c|c|c|c|c|c|c|c|c|c|c|c|}
\hline $\begin{array}{l}\text { storm } \\
\text { type }\end{array}$ & $\begin{array}{l}\text { JWD } \\
\text { data }\end{array}$ & \multicolumn{3}{|c|}{ scaling } & \multicolumn{3}{|c|}{ exponential DSD } & \multicolumn{4}{|c|}{ gamma DSD } \\
\hline \multirow{2}{*}{$\mathrm{C}$} & uncorrected & 0.186 & 0.174 & 0.998 & $5.47 \times 10^{3}$ & 3.90 & 0.273 & $3.73 \times 10^{4}$ & 5.66 & 2.11 & 0.592 \\
\hline & corrected & 0.170 & 0.178 & 0.997 & $5.27 \times 10^{3}$ & 3.87 & 0.180 & $2.88 \times 10^{4}$ & 5.43 & 1.88 & 0.265 \\
\hline \multirow{2}{*}{$\mathrm{T}$} & uncorrected & -0.061 & 0.227 & 1.000 & $1.19 \times 10^{4}$ & 4.61 & 0.609 & $2.40 \times 10^{6}$ & 9.54 & 5.00 & 0.812 \\
\hline & corrected & -0.062 & 0.227 & 0.996 & $1.17 \times 10^{4}$ & 4.59 & 0.709 & $7.13 \times 10^{5}$ & 8.39 & 3.87 & 0.770 \\
\hline$S$ & corrected & 0.071 & 0.199 & 0.999 & $2.30 \times 10^{3}$ & 3.24 & 0.658 & $5.88 \times 10^{3}$ & 4.13 & 1.29 & 0.645 \\
\hline
\end{tabular}

where $\Psi$ is a moment of $N_{V}(D)$, and the shape of $g(y)$ determines the shape of the DSD. It should be noted that the scaling exponents $\alpha$ and $\beta$ do not depend on the functional form of $g(y)$, as they are determined from the moments of $N_{V}(D)$. The reader is referred to Sempere Torres et al. (1994) and Uijlenhoet et al. (2003) for more details regarding this scalinglaw formalism. In this paper, we will consider the gamma distribution (Ulbrich, 1983) and the exponential distribution (Marshall and Palmer, 1948), which is a special case of the gamma distribution $(\mu=0)$

$N_{V}(D)=N_{0} D^{\mu} \mathrm{e}^{-\Lambda D}$.

The functional form of $g(y)$ from Eq. (17) that corresponds to this distribution is given by

$g(y)=\kappa y^{\mu} \mathrm{e}^{-\lambda y}$.

And the gamma-DSD parameters $N_{0}$ and $\Lambda$ can hence be expressed as

$$
\begin{aligned}
N_{0} & =\kappa \Psi^{\alpha-\mu \beta} \\
\Lambda & =\lambda \Psi^{-\beta} .
\end{aligned}
$$

For each of these periods in the event defined by Uijlenhoet et al. (2003) (convective, transition, and stratiform) the scaling-law formalism has been applied to derive parameters for both exponential and gamma DSDs. In these analyses (as in Uijlenhoet et al., 2003) the scaling is carried out with respect to the 3.67 th moment of $N_{V}(D), \hat{R}$. This moment is the rainfall intensity corresponding to the $v_{t}(D)$ relation by Atlas and Ulbrich (1977) (i.e. $v=3.778 D^{0.67}$ )

$\hat{R}=6 \pi \times 10^{-4} \times 3.778 \int_{0}^{\infty} D^{3.67} N_{V}(D) \mathrm{d} D$.

These scaling analyses are repeated here using the corrected DSDs, the results of which are compared to those obtained by Uijlenhoet et al. (2003).

Table 2 shows the results of these scaling analyses with the corrected JWD data. It is clear from this table that in terms of scaling exponents, the correction of the JWD data only has a significant effect on the stratiform part of the event. After correction, values of $\alpha$ decrease, whereas values of $\beta$ increase, indicating that rainfall variation is more controlled by variation in drop sizes rather than variation in numbers of drops. The exponential DSD parameters are seen to be only slightly affected by the correction. The most dramatic effect of correcting the JWD data for high-speed small drops is seen in the $\mu$-parameter of the gamma distributions. Especially for the transition and stratiform parts of the event the $\mu$-parameter decreases, indicating a more exponential shape of the scaled DSD. In the remainder of this paper, we assume that the parameters determined using the corrected JWD data are valid.

\section{Simulation of disdrometer measurements}

We investigate the effect of the observed deviation from theoretical fall velocities on bulk rainfall variables derived from measurements by different disdrometers, and on the derived power-law relations between such variables. The theoretical terminal fall velocities of drops are derived using the semi-empirical relations by Beard (1976), with a temperature of $288.16 \mathrm{~K}$ and a pressure of $101300 \mathrm{~Pa}$. For these simulations we assume that the raindrop size distributions (DSD) are gamma distributions (see Eq. (18)) with parameters from Table 2. Power-law relations between $R$ on the one hand and $Z, k_{27}$, and $k_{\text {opt }}$ on the other are fitted by linear regression of the logarithms of these variables. For these fits, 100 evenly spaced rainfall intensities between $1 \mathrm{~mm} \mathrm{~h}^{-1}$ and $100 \mathrm{~mm} \mathrm{~h}^{-1}$ are used.

\subsection{Joss-Waldvogel disdrometer}

We simulate JWD measurements using the methodology presented by Salles and Creutin (2003). Drop impacts on the styrofoam cone are computed from drop sizes and velocities (see Sect. 3). These drop impacts are then interpreted as if all drop velocities are the theoretical ones to obtain DSDs. The JWD measures $N_{A}(D)$, and hence $f_{A}(x)$ (see Eq. 1) will be 


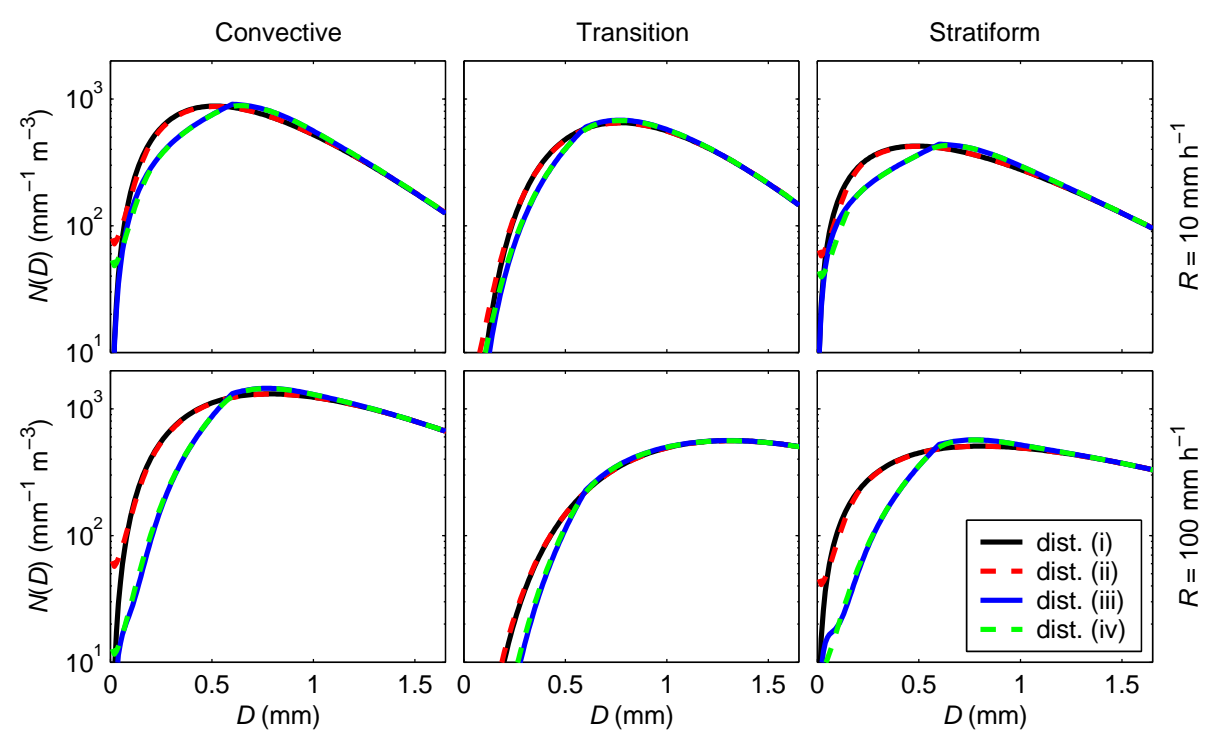

Fig. 4. Comparisons of DSDs derived using simulated measurements from a JWD, using underlying gamma DSDs for the three different types of rainfall. Results are shown for the four velocity ratio distributions listed in Table 1 . Analyses are shown for $R=10 \mathrm{~mm} \mathrm{~h}^{-1}$ (top panels) and $R=100 \mathrm{~mm} \mathrm{~h}^{-1}$ (bottom panels).

used as the distribution of velocity ratios for the simulations. For DSD simulation purposes, we will assume that the JWD has infinitesimal diameter classes. In reality, this is not the case, and the minimum diameter that can be detected by a JWD is $0.3 \mathrm{~mm}$. The true JWD diameter classes will be taken into account in the computation of bulk rainfall variables (i.e. $R, Z, k_{27}$, and $\left.k_{\mathrm{opt}}\right)$.

Figure 4 shows comparisons of DSDs derived from a JWD given the different distributions of velocity ratios (see Table 1) for gamma DSDs characteristic of convective, transition, and stratiform rain, and for rainfall intensities of $10 \mathrm{~mm} \mathrm{~h}^{-1}$ and $100 \mathrm{~mm} \mathrm{~h}^{-1}$ (note that below $R_{1}=2.5 \mathrm{~mm} \mathrm{~h}^{-1}$ there is no effect of high-velocity small raindrops). It is clear from this figure that the effect of high-velocity small drops is more pronounced than that of turbulence-induced variations in raindrop fall velocities. The expected decrease of numbers of small drops and increase of numbers of larger drops is clearly visible, especially for convective and stratiform rain. It should be noted, however, that for stratiform rain, intensities of $100 \mathrm{~mm} \mathrm{~h}^{-1}$ are highly unlikely. Nevertheless, the effect is clearly visible for stratiform rain at $10 \mathrm{~m} \mathrm{~h}^{-1}$.

\subsection{2-D Video Distrometer}

The 2-D Video Distrometer (2DVD, see e.g. Kruger and Krajewski, 2002) is capable of measuring drop diameters, drop fall velocities, and the shape of drops (often summarized as an aspect ratio). Because it uses two parallel sheets of light, it suffers from so-called mismatching (a drop seen by the first sheet of light is matched to a different drop seen by the sec- ond sheet of light), which yields errors in drop shapes and velocities. A method to overcome this problem is by using a drop size-velocity filter. In 2DVD data preprocessing, a filter is often employed such that the measured velocity of a given drop is within $40 \%$ of the theoretical terminal velocity of a drop with that diameter (Thurai and Bringi, 2005). Here we will investigate the effect of this filter on DSDs retrieved from simulated 2DVD measurements for the distributions of velocities given in Sect. 2. We will not consider the effect of drop size classes for the 2DVD, as these are only $0.01 \mathrm{~mm}$ wide. Like the JWD, the 2DVD measures drops arriving at a surface, and hence $f_{A}(x)$ (Eq. 1) will be used as the distribution of velocity ratios for the simulations of 2DVD measurements.

Figure 5 shows comparisons of DSDs derived from a 2DVD given the different distributions of velocity ratios (see Table 1) for gamma DSDs characteristic of convective, transition, and stratiform rain, and for rainfall intensities of $10 \mathrm{~mm} \mathrm{~h}^{-1}$ and $100 \mathrm{~mm} \mathrm{~h}^{-1}$. In contrast to the JWD, as expected, the effect of high-velocity small drops is limited to a decrease of the number of drops smaller than $D_{1}=0.6 \mathrm{~mm}$. As for the JWD, the effect is mostly visible in convective and stratiform rain. This is to be expected as the gamma distribution characteristic for transition rainfall has a high $\mu$, indicating a relatively small number of small drops. Another aspect of these graphs is that the effect of turbulenceinduced variations in fall velocities becomes apparent, and is more important than the effect of high-velocity small drops at lower rainfall intensities. This can be concluded from the fact that there is a difference between the lines corresponding to distributions where turbulence is ((ii) and (iv)) and is 

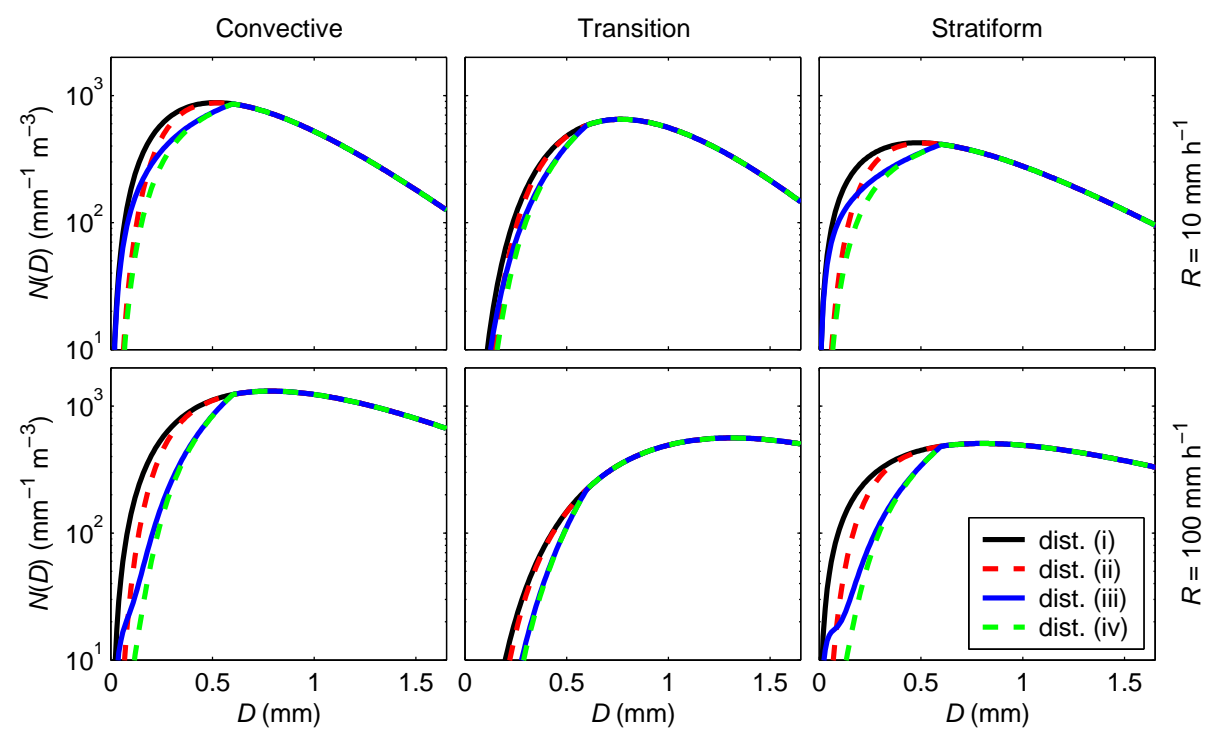

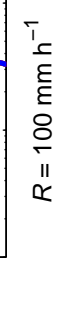

Fig. 5. As Fig. 4, but for simulated 2DVD measurements.
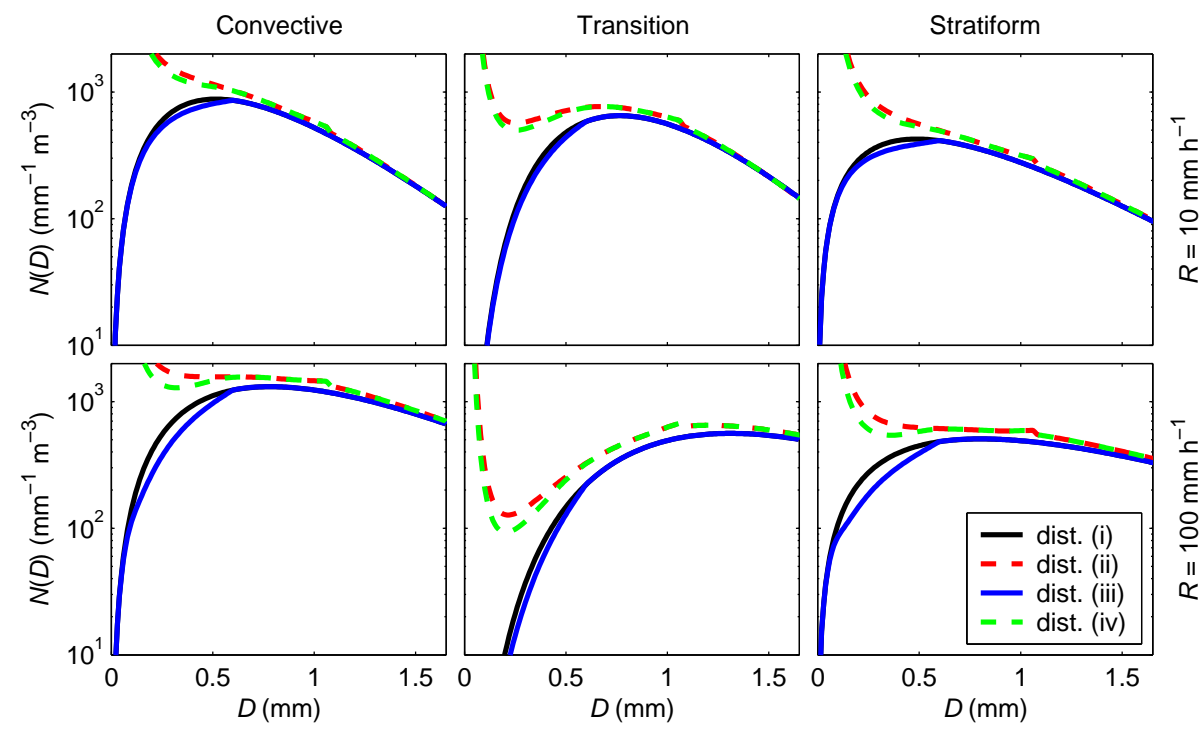

Fig. 6. As Fig. 4, but for simulated measurements from vertically-pointing Doppler radar.

not ((i) and (iii), respectively) taken into account. Especially at $10 \mathrm{~mm} \mathrm{~h}^{-1}$ (top panels of Fig. 5), this difference is larger than the difference between the lines corresponding to distributions where high speed small drops are ((iii) and (iv)) and are not ((i) and (ii), respectively) taken into account (see also Table 1).

\subsection{Doppler spectra}

Doppler radars can be used to estimate drop size distributions (e.g. Sheppard, 1990; Doviak and Zrnic, 1993). A Doppler spectrum as measured by a vertically-pointing radar is the re- flectivity per vertical velocity class. DSDs are derived from Doppler spectra by assuming a monotonic relation between drop diameter and fall velocity. The modified fall velocities suggested by Montero-Martínez et al. (2009) will influence this Doppler spectrum through shifting some of the power from the low-velocity classes to higher velocity classes. We will analyse the effect of this shift in power, but we expect it to be minor (as was also stated by Montero-Martínez et al., 2009). Because radars sample a volume of air, $f_{V}(x)$ (Eq. (3)) will be used as the distribution of velocity ratios for the simulation of Doppler spectra. 

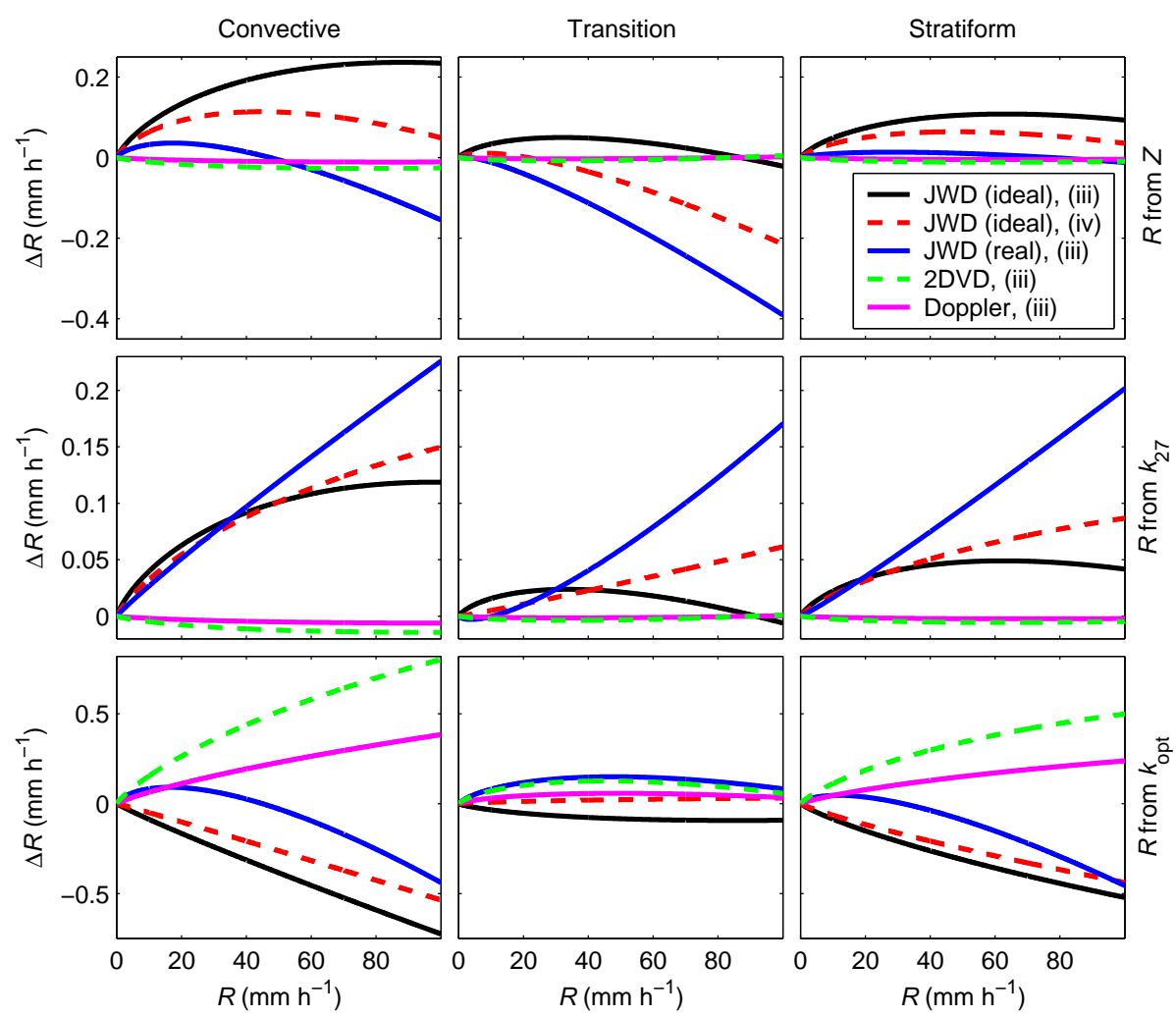

Fig. 7. Errors in the rainfall intensity $(\Delta R)$ resulting from the use of an inappropriate retrieval relation (see Table 3 ) as a function of true $R$ for $Z-R$ relations (top panels), $k_{27}-R$ relations (middle panels), and $k_{\mathrm{opt}}-R$ relations (bottom panels). Results for relations based on DSDs from a JWD with infinitesimal diameter classes (ideal), a JWD with real diameter classes, a 2DVD and a vertically-pointing Doppler radar are shown. The effect of turbulence-induced velocity variations is also shown for the ideal JWD (distribution (iv)).

Figure 6 shows comparisons of DSDs derived from simulated Doppler spectra from a vertically-pointing radar given the different distributions of velocity ratios (see Table 1) for gamma DSDs characteristic of convective, transition, and stratiform rain, and for rainfall intensities of $10 \mathrm{~mm} \mathrm{~h}^{-1}$ and $100 \mathrm{~mm} \mathrm{~h}^{-1}$. It is immediately clear from these graphs that the effect of high-velocity small drops is small. But the most striking aspect of these graphs is the very large effect of turbulence-induced velocity variations. Even very lowintensity turbulence $\left(\sigma_{v}=0.3 \mathrm{~m} \mathrm{~s}^{-1}\right.$, see Sect. 2) causes large overestimations of the DSD for drops smaller than $1 \mathrm{~mm}$. It can hence be safely stated that for Doppler spectra-derived DSDs the effect of high-velocity small drops is negligible compared to the effect of turbulence-induced velocity variations.

\section{Effect on derived retrieval relations}

Table 3 shows the coefficients $a$ and exponents $b$ of powerlaw relations between $R$ and the remote sensing bulk rainfall variables $Z, k_{27}$, and $k_{\text {opt }}$. Only the coefficients of the derived $Z-R$ power-law relation are affected by high-velocity small drops. This effect is of the same order of magnitude as the effect of the finite classes of the JWD for $Z-R$ relations, and even larger for $k_{\mathrm{opt}}-R$ power-law relations. As expected, the effect on all power-law retrieval relations based on 2DVDderived DSDs is minor. The variation in power-law relations between $k_{27}$ and $R$ is limited. This can be explained by the fact that these relations have little dependence on the shape of the underlying DSD (Atlas and Ulbrich, 1977; Leijnse et al., 2007b), as opposed to $Z-R$ and $k_{\mathrm{opt}}-R$ relations (Leijnse et al., 2007a; Uijlenhoet et al., 2010). As was shown in Sect. 4.3, turbulence-induced velocity variations can play a role, and greatly affect the $k_{\mathrm{opt}}-R$ relations derived from vertically-pointing Doppler radar.

Figure 7 shows the errors in the retrieved $R$ that occur because of the use of an inappropriate relation

$\Delta R=\left(\frac{X}{a_{X}}\right)^{1 / b_{X}}-\left(\frac{X}{a_{X, \text { true }}}\right)^{1 / b_{X, \text { true }}}$,

where $X$ can be $Z, k_{27}$, or $k_{\mathrm{opt}}$, and $a_{X}$ and $b_{X}$ are values from Table 3. This figure shows the effect of errors in $Z-R$, $k_{27}-R$, and $k_{\mathrm{opt}}-R$ relations derived from ideal (i.e. infinitesimal diameter classes) and real JWD measurements, as 
Table 3. Coefficients $a$ and exponents $b$ of fitted $Z-R, k_{27}-R$, and $k_{\mathrm{opt}}-R$ relations for different simulated DSDs (see Table 1 for the meaning of (i)-(iv)). Fits were carried out by linear regression of $\log (Z), \log \left(k_{27}\right)$, and $\log \left(k_{\mathrm{opt}}\right)$ on $\log (R)$.

\begin{tabular}{|c|c|c|c|c|c|c|c|c|}
\hline storm type & DSD type & distribution & $a_{Z}$ & $b_{Z}$ & $a_{k_{27}}$ & $b_{k_{27}}$ & $a_{k_{\mathrm{opt}}}$ & $b_{k_{\mathrm{opt}}}$ \\
\hline \multirow{8}{*}{ Convective } & \multirow{3}{*}{ JWD (ideal) } & (ii) & 252 & 1.41 & 0.135 & 1.06 & 0.96 & 0.713 \\
\hline & & (iii) & 246 & 1.41 & 0.134 & 1.07 & 0.97 & 0.712 \\
\hline & & (iv) & 247 & 1.41 & 0.134 & 1.07 & 0.97 & 0.713 \\
\hline & \multirow{2}{*}{ JWD (real) } & (i) & 254 & 1.41 & 0.135 & 1.06 & 0.94 & 0.717 \\
\hline & & (iv) & 249 & 1.41 & 0.135 & 1.06 & 0.95 & 0.717 \\
\hline & \multirow{3}{*}{ 2DVD } & (ii) & 252 & 1.41 & 0.135 & 1.07 & 0.96 & 0.714 \\
\hline & & (iii) & 252 & 1.41 & 0.135 & 1.07 & 0.95 & 0.715 \\
\hline & & (iv) & 252 & 1.41 & 0.135 & 1.07 & 0.95 & 0.716 \\
\hline \multirow{10}{*}{ Transition } & \multicolumn{2}{|c|}{ true } & 155 & 1.54 & 0.124 & 1.09 & 1.17 & 0.630 \\
\hline & \multirow{3}{*}{ JWD (ideal) } & (ii) & 156 & 1.54 & 0.124 & 1.09 & 1.16 & 0.630 \\
\hline & & (iii) & 153 & 1.54 & 0.124 & 1.09 & 1.17 & 0.629 \\
\hline & & (iv) & 154 & 1.54 & 0.124 & 1.09 & 1.17 & 0.630 \\
\hline & \multirow{4}{*}{ JWD (real) } & (i) & 156 & 1.54 & 0.125 & 1.09 & 1.15 & 0.632 \\
\hline & & (ii) & 157 & 1.54 & 0.125 & 1.09 & 1.15 & 0.633 \\
\hline & & (iii) & 155 & 1.54 & 0.124 & 1.09 & 1.16 & 0.632 \\
\hline & & (iv) & 155 & 1.54 & 0.125 & 1.09 & 1.15 & 0.632 \\
\hline & \multirow[b]{2}{*}{ 2DVD } & (ii) & 155 & 1.54 & 0.124 & 1.09 & 1.16 & 0.630 \\
\hline & & (iii) & 155 & 1.54 & 0.124 & 1.09 & 1.16 & 0.631 \\
\hline \multirow{12}{*}{ Stratiform } & \multirow[t]{2}{*}{ JWD (ideal) } & (iii) & 420 & 1.41 & 0.157 & 1.03 & 0.73 & 0.700 \\
\hline & & (iv) & 421 & 1.41 & 0.157 & 1.03 & 0.73 & 0.700 \\
\hline & \multirow{4}{*}{ JWD (real) } & (i) & 429 & 1.41 & 0.158 & 1.03 & 0.71 & 0.705 \\
\hline & & (ii) & 431 & 1.41 & 0.158 & 1.03 & 0.71 & 0.705 \\
\hline & & (iii) & 424 & 1.41 & 0.158 & 1.03 & 0.72 & 0.703 \\
\hline & & (iv) & 425 & 1.41 & 0.158 & 1.03 & 0.72 & 0.704 \\
\hline & \multirow{3}{*}{ 2DVD } & (ii) & 425 & 1.41 & 0.158 & 1.03 & 0.72 & 0.702 \\
\hline & & (iii) & 426 & 1.41 & 0.158 & 1.03 & 0.72 & 0.703 \\
\hline & & (iv) & 426 & 1.41 & 0.158 & 1.03 & 0.71 & 0.704 \\
\hline & \multirow{3}{*}{ Doppler } & (ii) & 421 & 1.38 & 0.155 & 1.04 & 1.53 & 0.549 \\
\hline & & (iii) & 425 & 1.41 & 0.158 & 1.03 & 0.72 & 0.701 \\
\hline & & (iv) & 422 & 1.38 & 0.155 & 1.04 & 1.50 & 0.550 \\
\hline
\end{tabular}


well from a 2DVD and a vertically-pointing Doppler radar. Essentially, this figure is a summary of the effect of the different retrieval relations of Table 3. The effects of the finite drop diameter classes, of high-velocity small drops, and of turbulence-induced velocity variations are of the same order of magnitude for the JWD. The absolute magnitude of the errors caused by the use of erroneous retrieval relations is very small, and will be completely masked by other sources of error in any practical situation (Krajewski and Smith, 2002; Salles and Creutin, 2003; Uijlenhoet et al., 2003; Leijnse et al., 2007b, 2008; Uijlenhoet et al., 2010). The large effect of the turbulence-induced velocity variations on retrieval relations based on Doppler spectra apparent from Table 3 is not shown in Fig. 7, because the maximum errors for these relations are between $10 \mathrm{~mm} \mathrm{~h}^{-1}$ and $15 \mathrm{~mm} \mathrm{~h}^{-1}$ (which is orders of magnitude larger than the other errors presented in this graph).

\section{Conclusions}

It has recently been shown by Montero-Martínez et al. (2009) that small raindrops may fall significantly faster than their theoretical terminal fall velocities because small drops are often the result of the breakup of fast-moving large raindrops. We have fitted lognormal distributions of velocity ratios to the data presented in Fig. 2 of Montero-Martínez et al. (2009). The parameters of these distributions depend on both drop size and rainfall intensity. From these distributions a correction method for Joss-Waldvogel disdrometer data has been developed. This correction method has been applied to JWD data for a squall line (Uijlenhoet et al., 2003). The scaling-law formalism was applied to these corrected JWD data and compared with results from Uijlenhoet et al. (2003), who carried out the same analyses. Both exponential and gamma DSDs were fitted in this framework, for three types of rainfall that occurred within this event. The correction is seen to yield different results, depending on the precipitation type. Especially the $\mu$-parameter of fitted gamma DSDs is affected by the correction.

Using the derived gamma DSDs for the three different precipitation types, disdrometer measurements and Doppler spectra have been simulated to quantify the effect of the breakup-induced deviations from theoretical velocities on retrieved raindrop size distributions. This effect has only a minor influence on Joss-Waldvogel disdrometer, 2-D Video Distrometer, and Doppler spectra measurements. The resulting effect on retrieved $Z-R, k_{27}-R$, and $k_{\mathrm{opt}}-R$ power-law relations is also minor, and much smaller than that related to other issues such as systematic deviations from the theoretical $v_{t}(D)$-relation over the entire range of drop sizes (Salles and Creutin, 2003), drop sampling effects (Tokay et al., 2005; Uijlenhoet et al., 2006), or other sources of error (Krajewski and Smith, 2002; Leijnse et al., 2007b, 2008; Uijlenhoet et al., 2010). For the JWD, the effect of high-velocity small raindrops is of the same order of magnitude as that of the finite drop size classes, and that of the turbulence-induced velocity variations. For Doppler spectra-derived retrieval relations, the effect of turbulence is much larger than that of high-velocity small drops. Furthermore, the errors in radar rainfall estimates caused by the slight differences in $Z-R$ relations are very small compared to the errors caused by DSD variability (Uijlenhoet et al., 2003). Therefore it can be concluded that for remote sensing applications, the effect of high-velocity small drops is negligible.

It would be interesting to repeat the analyses of MonteroMartínez et al. (2009) in different places, as raindrops falling in Mexico City may differ from those elsewhere. It could be argued that the effect shown by Montero-Martínez et al. (2009) might be enhanced by the fact that pollution alters the surface tension of water, resulting in more frequent breakup of drops (e.g. Pruppacher and Klett, 1997).

Acknowledgements. The authors would like to thank Matthias Steiner (NCAR) for collecting and providing the Goodwin Creek dataset. Hidde Leijnse was financially supported by The Netherlands Organization for Scientific Research (NWO) through a postdoctoral grant in the framework of the Water programme.

Edited by: G. Roberts

\section{References}

Atlas, D. and Ulbrich, C. W.: Path- and area-integrated rainfall measurement by microwave attenuation in the $1-3 \mathrm{~cm}$ band, J. Appl. Meteorol., 16, 1322-1331, 1977.

Battan, L. J.: Radar Observation of the Atmosphere, University of Chicago Press, 324 pp., 1973.

Beard, K. V.: Terminal velocity and shape of cloud and precipitation drops aloft, J. Atmos. Sci., 33, 851-864, 1976.

Doviak, R. J. and Zrnic, D. S.: Doppler radar and weather observations, Academic Press, 2nd edn., 1993.

Joss, J. and Waldvogel, A.: Ein Spectrograph für Niederschlagstropfen mit automatischer Auswertung, Pure Appl. Geophys., 69, 240-246, 1967.

Joss, J. and Waldvogel, A.: Comments on "Some observations on the Joss-Waldvogel rainfall disdrometer”, J. Appl. Meteorol., 16, 112-113, 1977.

Krajewski, W. F. and Smith, J. A.: Radar Hydrology: rainfall estimation, Adv. Water Resour., 25, 1387-1394, doi:10.1016/S03091708(02)00062-3, 2002.

Kruger, A. and Krajewski, W. F.: Two-Dimensional Video Disdrometer: A Description, J. Atmos. Oceanic Technol., 19, 602617, 2002.

Leijnse, H., Uijlenhoet, R., and Stricker, J. N. M.: Rainfall measurement using radio links from cellular communication networks, Water Resour. Res., 43, W03201, doi:10.1029/2006WR005631, 2007a.

Leijnse, H., Uijlenhoet, R., and Stricker, J. N. M.: Hydrometeorological application of a microwave link: 2. Precipitation, Water Resour. Res., 43, W04417, doi:10.1029/2006WR004989, 2007b.

Leijnse, H., Uijlenhoet, R., and Stricker, J. N. M.: Microwave link rainfall estimation: Effects of link length and 
frequency, temporal sampling, power resolution, and wet antenna attenuation, Adv. Water Resour., 31, 1481-1493, doi:10.1016/j.advwatres.2008.03.004, 2008.

Löffler-Mang, M., Kunz, M., and Schmid, W.: On the performance of a low-cost K-Band Doppler radar for quantitative rain measurements, J. Atmos. Ocean. Technol., 16, 379-387, 1999.

Marshall, J. S. and Palmer, W. M.: The distribution of raindrops with size, J. Meteorol., 5, 165-166, 1948.

Mishchenko, M. I.: Calculation of the amplitude matrix for a nonspherical particle in a fixed orientation, Appl. Opt., 39, 10261031, 2000.

Montero-Martínez, G., Kostinski, A. B., Shaw, R. A., and GarcíaGarcía, F.: Do all raindrops fall at terminal speed?, Geophys. Res. Lett., 36, L11818, doi:10.1029/2008GL037111, 2009.

Mood, A. M., Graybill, F. A., and Boes, D. C.: Introduction to the Theory of Statistics, McGraw-Hill, 3rd edn., 480 pp., 1974.

Pruppacher, H. R. and Klett, J. D.: Microphysics of Clouds and Precipitation, Kluwer Academic Publishers, 2nd edn., 954 pp., 1997.

Salles, C. and Creutin, J.-D.: Instrumental uncertainties in $Z-R$ relationships and raindrop fall velocities, J. Appl. Meteorol., 42, 279-290, 2003.

Sempere Torres, D., Porrà, J. M., and Creutin, J.-D.: A general formulation for raindrop size distribution, J. Appl. Meteorol., 33, 1494-1502, 1994.
Sheppard, B. E.: Measurement of raindrop size distributions using a small Doppler radar, J. Atmos. Ocean. Technol., 7, 255-268, 1990.

Thurai, M. and Bringi, V. N.: Drop axis ratios from a 2D Video Disdrometer, J. Atmos. Oceanic Technol., 22, 966-978, 2005.

Tokay, A., Bashor, P. G., and Wolff, K. R.: Error characteristics of rainfall measurements by collocated Joss-Waldvogel disdrometers, J. Atmos. Ocean. Technol., 22, 513-527, 2005.

Uijlenhoet, R. and Stricker, J. N. M.: A consistent rainfall parameterization based on the exponential raindrop size distribution, J. Hydrol., 218, 101-127, 1999.

Uijlenhoet, R., Steiner, M., and Smith, J. A.: Variability of raindrop size distributions in a squall line and implications for radar rainfall estimation, J. Hydrometeorol., 4, 43-61, 2003.

Uijlenhoet, R., Porrà, J. M., Sempere Torres, D., and Creutin, J.-D.: Analytical solutions to sampling effects in drop size distribution measurements during stationary rainfall: Estimation of bulk rainfall variables, J. Hydrol., 328, 65-82, 2006.

Uijlenhoet, R., Cohard, J.-M., and Gosset, M.: Path-average rainfall estimation from optical extinction measurements using a largeaperture scintillometer, J. Hydrometeorol., in preparation, 2010.

Ulbrich, C. W.: Natural variations in the analytical form of the raindrop size distribution, J. Appl. Meteorol., 22, 1764-1775, 1983. 